Document downloaded from:

http://hdl.handle.net/10251/35189

This paper must be cited as:

Abad Rodriguez, MF.; Gasso Matoses, MT.; Torregrosa Sánchez, JR. (2011). Some results about inverse-positive matrices. Applied Mathematics and Computation. 218(1):130-139. doi:10.1016/j.amc.2011.05.063.

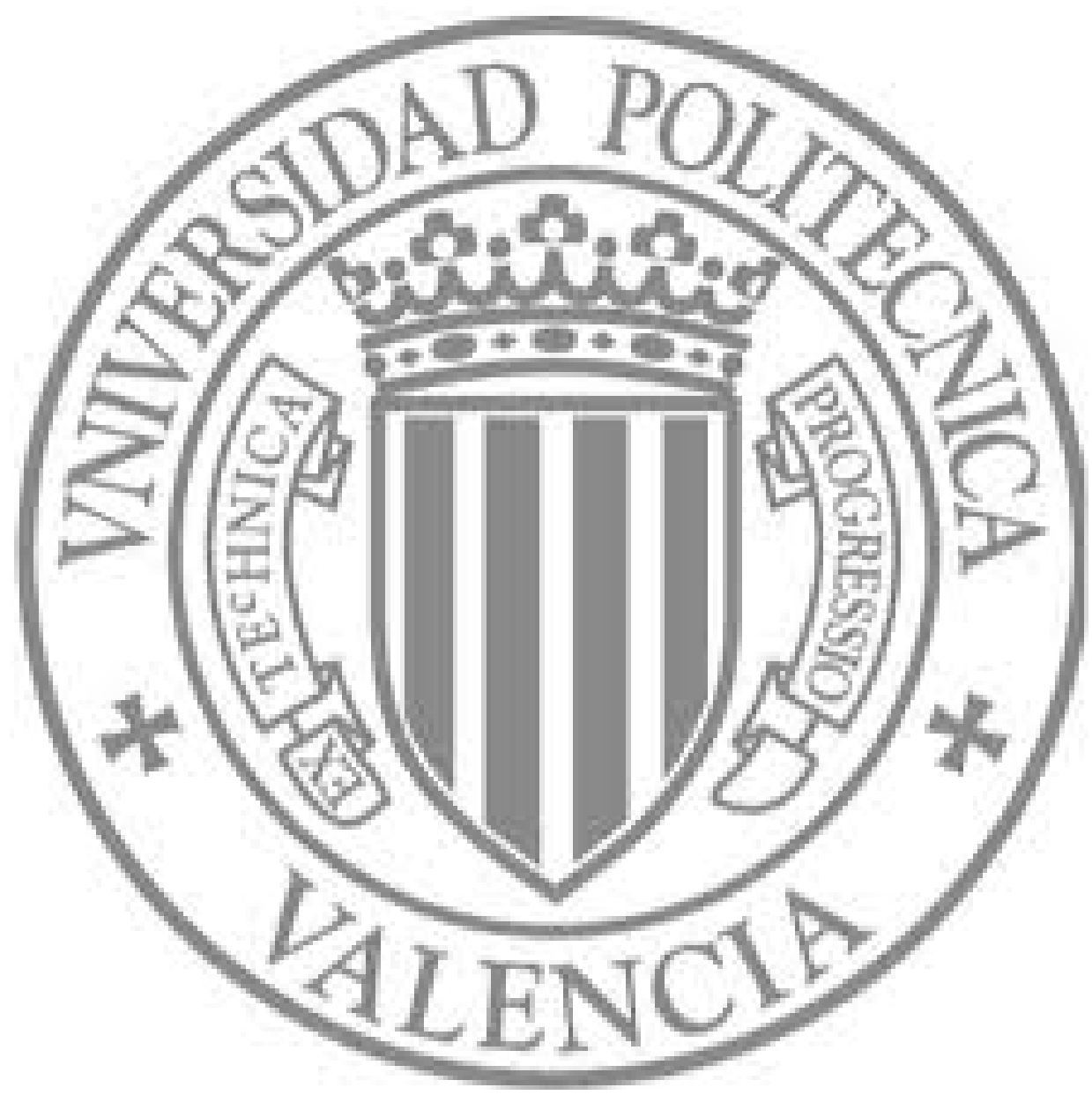

The final publication is available at

http://dx.doi.org/10.1016/j.amc.2011.05.063

Copyright Elsevier 


\title{
Some results about inverse-positive matrices *
}

\author{
Manuel F. Abad*, María T. Gassó, Juan R. Torregrosa. \\ Instituto de Matemática Multidisciplinar, \\ Universitat Politècnica de València, \\ Camino de Vera, s/n, 46022 \\ Valencia, Spain.
}

\begin{abstract}
A nonsingular real matrix $A$ is said to be inverse-positive if all the elements of its inverse are nonnegative. This class of matrices contains the $M$-matrices, from which inherit some of their properties and applications, especially in Economy and in the description of iterative methods for solving nonlinear systems. In this paper we present some new characterizations for inverse-positive matrices and we analyze when this concept is preserved by the sub-direct sum of matrices.
\end{abstract}

Key words: Inverse-positive matrix, $M$-matrix, Sub-direct sum. 2010 MSC: 15A09, 15B48.

\section{Introduction}

A nonsingular real matrix $A=\left(a_{i j}\right)$ is said to be inverse-positive if all the elements of its inverse are nonnegative. An inverse-positive matrix being also a $Z$-matrix, is a nonsingular $M$-matrix, so the class of inverse-positive matrices contains the nonsingular $M$-matrices, which have been widely studied and whose applications, for example, in iterative methods, dynamic systems, economics, mathematical programming, etc, are well known. Of course, not

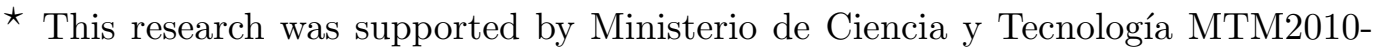
18539

* Corresponding author

Email addresses: maabrod@mat.upv.es (Manuel F. Abad), mgasso@mat.upv.es (María T. Gassó), jrtorre@mat.upv.es (Juan R. Torregrosa.). 
every inverse-positive matrix is an $M$-matrix. For instance,

$$
A=\left(\begin{array}{rr}
0 & 2 \\
3 & -1
\end{array}\right),
$$

is an inverse-positive matrix that is not an $M$-matrix.

The inverse-positivity is preserved by multiplication and hence by left or right positive diagonal multiplication, positive diagonal similarity and permutation similarity.

The problem of characterizing inverse-positive matrices has been extensively dealt with in the literature (see, for example [1]). Besides, M. Fiedler in [2] showed several characterizations of sign patterns of inverse positive matrices and J.E. Peris [3] provides a characterization of inverse-positive matrices using positive splittings. More recently, T. Fujimoto et al. presented in [4] a characterization of inverse-positive matrices concerning the Hawkins-Simon condition, so called in economics, and the Le Chatelier-Braun principle in thermodynamics (see [5]).

The inverse-positivity of real square matrices plays an important role in different areas of science and engineering and has been analyzed in several contexts. For example, in [6] we can see the description of particular iterative methods for solving linear systems, when the matrix is inverse-positive. R. Precup [7] obtains positive nontrivial solutions for a class of semilinear elliptic variational systems by using the technique of inverse-positive matrices. In the recurrence relation of the Chebyshev polynomials of second kind appear several inversepositive matrices which have been studied by C.M. da Fonseca in [8].

There exists a special case of matrices that, as we can see in [9], very often occur in relation to Leontief model of circulating capital without joint production. For instance, matrices that for size $5 \times 5$ have the form

$$
A=\left(\begin{array}{rrrrr}
1 & -a & 1 & -a & 1 \\
1 & 1 & -a & 1 & -a \\
-a & 1 & 1 & -a & 1 \\
1 & -a & 1 & 1 & -a \\
-a & 1 & -a & 1 & 1
\end{array}\right)
$$

where $a$ is a real parameter with economic interpretation. When $A$ is an $n \times n$ real matrix pertaining this special case with $n$ odd, $n=2 k+1$, it is not difficult to prove that $A$ is an inverse-positive matrix if and only if $1 \leq a<\left(1+\frac{1}{k}\right)$. 
The sub-direct sum of matrices is a generalization of the usual sum of matrices. This concept was introduced by C. Johnson and S. Fallat in [10] and arises naturally in matrix completions and overlapping subdomains in domain decomposition methods, among other contexts. It also appears in many variants of additive Schwartz preconditioning, and when analyzing additive Schwartz methods for Markov chains.

Domain decomposition methods, are widely used for the numerical solution of partial differential equations (see, for example [11]). In [12] the authors analyze the convergence and properties for several variants of these methods for nonsingular inverse-positive matrices.

The paper is structured as follows. In Section 2 we present some conditions in order to obtain new characterizations for inverse-positive matrices. In Section 3 we study the question of when the sub-direct sum of two inverse-positive matrices is an inverse-positive matrix, and conversely, when an inverse-positive matrix can be expressed as the sub-direct sum of two inverse-positive matrices.

We explain some notations used in this paper. $(A)_{i j}$ denotes the $(i, j)$ entry of $A$. The symbol $(A)_{j}$ means the $j$ th column of $A$ and $\operatorname{det}(A(i \mid j))$ means the determinant of the matrix that results by deleting row $i$ and column $j$ of matrix $A$.

\section{Characterization of inverse-positive matrices}

In this section we present some new characterizations of inverse-positive matrices.

Let $x$ be a vector in $\mathbb{R}^{n}$. In this paper we write $x \geq 0$ when all the components of $x$ are nonnegative, $x>0$ when all the components of $x$ are nonnegative but not all equal to zero, and $x \gg 0$ when all the components of $x$ are positive.

Let $A$ be an $n \times n$ real matrix. Consider a nonempty subset $S$ of $N=$ $\{1,2, \ldots, n\}$ and $T=S^{c}$, the complement of $S$ with respect to $N$. Now, we consider the following property

\section{Property}

Given $x \geq 0$ and $A x=\left(b_{1}, b_{2}, \ldots, b_{n}\right)^{T}$,

$$
\text { If } b_{j}>0, \forall j \in S \text { and } b_{j}=0, \forall j \in T \text {, then } x \gg 0 \text {. }
$$

Given a subset $S$ of $N$, let $\mathcal{C}_{\mathcal{S}}$ be the class of $n \times n$ real matrices that satisfy 
(1) with respect to $S$. We can establish the following result.

Proposition 1 Let $A$ be a nonsingular real matrix of size $n \times n$. Then, $A \in \mathcal{C}_{\mathcal{S}}$ if and only if $\left(A^{-1}\right)_{j}>0, \forall j \in S$ and given $i \in N, \exists j \in S$ such that $\left(A^{-1}\right)_{i j}>0$.

Proof. Let $A$ be an element of $\mathcal{C}_{\mathcal{S}}$ with $A^{-1}=\left(c_{i j}\right)$ and $S=\left\{i_{1}, i_{2}, \ldots, i_{k}\right\}$ a subset of $N$. Suppose that there exists an index $i_{p} \in S$ such that $\left(A^{-1}\right)_{i_{p}}$ has a negative component (it is not possible to be the null vector because matrix $A$ is nonsingular).

Consider a vector $b=\left(b_{1}, b_{2}, \ldots, b_{n}\right)^{T}$ such that $b_{i}=0$ for $i \in T$ and $b_{i}>0$ for $i \in S$. Then, we can choose $b_{i_{p}}$ sufficiently large such that $x=A^{-1} b \geq 0$ and its $i_{p}$ th component zero, which is a contradiction with (1).

Besides, suppose that there exists an index $i_{0} \in \mathbb{N}$ such that $c_{i_{0} j}=0, \forall j \in S$. Consider a vector $b=\left(b_{1}, b_{2}, \ldots, b_{n}\right)^{T}$ with $b_{i}=0$ for $i \in T$ and $b_{i}>0$ for $i \in S$, and such that $x=A^{-1} b \geq 0$. Then, vector $x$ has at least its $i_{0}$ th component equal to zero, which is a contradiction with (1).

Conversely, suppose that there exists $x \geq 0$ satisfying (1) with some component equal to zero, for example $x_{p}=0$. Consider the system $A x=\left(b_{1}, b_{2}, \ldots, b_{n}\right)^{T}$, with $b_{i}>0$ for $i \in S$ and $b_{i}=0$ for $i \in T$. The $p$ th component of the solution of this system is $x_{p}=c_{p_{1}} b_{1}+c_{p_{2}} b_{2}+\ldots+c_{p_{n}} b_{n}=c_{p, i_{1}} b_{i_{1}}+\ldots+c_{p, i_{k}} b_{i_{k}}=0$. But, $x_{p}$ only can be zero if $c_{p, i_{j}}=0$, for $j=1,2, \ldots, k$, which is a contradiction with given $i \in N, \exists j \in S$ such that $\left(A^{-1}\right)_{i j}>0$.

Notice that when $S=N$, Proposition 1 is a characterization of inverse-positive matrices.

The next characterization of inverse-positive matrices is related to the existence of a positive solution of a linear system with positive independent term.

Theorem 1 An $n \times n$ real matrix $A$ is inverse-positive if and only if for all $b \gg 0$, there exists $x \gg 0$ such that $A x=b$.

Proof. If $A$ is inverse-positive, given a vector $b \gg 0$, then $x=A^{-1} b \gg 0$. Conversely, first we are going to prove, by using reduction to the absurd, that matrix $A$ is nonsingular. Let us suppose that $\operatorname{rank}(A)=r,(r<n)$, and we may assume, without loss of generality, that the first $r$ columns of $A$, $c_{1}, c_{2}, \ldots, c_{r}$, are linearly independent.

Let $b$ be a vector such that $b \gg 0$ and $c_{1}, c_{2}, \ldots, c_{r}, b$ are linearly independent vectors. So, system $A x=b$ has no solutions, which is a contradiction.

Now, we are going to prove that $A^{-1}=\left(c_{i j}\right)$ is nonnegative. Consider a vector 
$b \in \mathbb{R}^{n}, b \gg 0$, and suppose that there exists a pair $\left(i_{0}, j_{0}\right)$ such that $c_{i_{0}, j_{0}}<0$. As $x=A^{-1} b$,

$$
x_{i_{0}}=\sum_{j=1}^{n} c_{i_{0}, j} b_{j}=c_{i_{0}, 1} b_{1}+c_{i_{0}, 2} b_{2}+\ldots+c_{i_{0}, n} b_{n} .
$$

Suppose that we choose a vector $b=\left(b_{1}, b_{2}, \ldots, b_{n}\right)^{T}$ satisfying

$$
\left|c_{i_{0}, j_{0}} b_{j_{0}}\right| \geq \sum_{j=1, j \neq j_{0}}^{n} c_{i_{0}, j} b_{j} .
$$

Therefore $x_{i_{0}}$ is negative, which is a contradiction, so matrix $A$ is inversepositive.

In the last characterization we obtain a relation between inverse-positive and monotone matrices.

We recall that an $n \times n$ real matrix $A$ is said to be monotone if

$$
A x \geq 0 \rightarrow x \geq 0, \text { for all } x \in \mathbb{R}^{n} .
$$

Proposition 2 Let $A$ be an $n \times n$ real matrix. Then $A$ is inverse-positive if and only if $A$ is monotone.

Proof. If $A$ is inverse-positive, then it is obviously monotone.

Conversely, first we are going to prove that $A$ is nonsingular. As $A$ is monotone, from $A x=0$ and $A(-x)=0$ we obtain $x \geq 0$ and $x \leq 0$, respectively, that is, $x=0$. So the system $A x=0$ has only the trivial solution and therefore $A$ is nonsingular.

On the other hand, let $\left\{e_{1}, e_{2}, \ldots, e_{n}\right\}$ be the canonical base in $\mathbb{R}^{n}$. As $A\left(A^{-1} e_{i}\right) \geq$ 0 , we have $\left(A^{-1} e_{i}\right) \geq 0, i=1, \ldots, n$, so $A^{-1} \geq 0$.

\section{Sub-direct sum of inverse-positive matrices}

As we have said in the introduction, the sub-direct sum was introduced by Johnson and Fallat in [10], where many of its properties were analyzed.

Definition 1 Let $A, B$ be square matrices of size $n_{1}$ and $n_{2}$, respectively, and let $k$ be an integer such that $1 \leq k \leq \min \left\{n_{1}, n_{2}\right\}$. Suppose that $A$ and $B$ are 
partitioned as follows

$$
A=\left(\begin{array}{ll}
A_{11} & A_{12} \\
A_{21} & A_{22}
\end{array}\right), \quad B=\left(\begin{array}{ll}
B_{11} & B_{12} \\
B_{21} & B_{22}
\end{array}\right),
$$

where $A_{22}$ and $B_{11}$ are square matrices of size $k \times k$. Then, the sub-direct sum of size $k$ of $A$ and $B$, denoted by $C=A \oplus_{k} B$, is the matrix of size $n_{1}+n_{2}-k$

$$
C=\left(\begin{array}{ccc}
A_{11} & A_{12} & 0 \\
A_{21} & A_{22}+B_{11} & B_{12} \\
0 & B_{21} & B_{22}
\end{array}\right)
$$

The sub-direct sum of two inverse-positive matrices is not in general an inversepositive matrix, as we can see in the following example.

Example 1 Consider the inverse-positive matrices

$$
A=\left(\begin{array}{rr|rr}
-1 & 2 & 0 & 0 \\
3-1 & 0 & 0 \\
\hline-1 & -1 & 6 & -4 \\
-1 & -1 & -1 & 1
\end{array}\right) \quad \text { and } B=\left(\begin{array}{rr|rr}
-2 & 1 & 0 & 0 \\
8 & -1 & 0 & 0 \\
\hline-1-1 & -1 & 2 \\
-1 & -1 & 3 & -1
\end{array}\right)
$$

It is not difficult to see that matrix

$$
C=A \oplus_{2} B=\left(\begin{array}{rr|rr|rr}
-1 & 2 & 0 & 0 & 0 & 0 \\
3 & -1 & 0 & 0 & 0 & 0 \\
\hline-1 & -1 & 4 & -3 & 0 & 0 \\
-1 & -1 & 7 & 0 & 0 & 0 \\
\hline 0 & 0 & -1 & -1 & -1 & 2 \\
0 & 0 & -1 & -1 & 3 & -1
\end{array}\right)
$$

is not inverse-positive.

In this section we study the conditions under which the sub-direct sum of inverse-positive matrices lies in the class, and it is appropriate to consider $k=1$ and $k>1$ separately.

We also study the following question: 
If $C$ is an inverse-positive matrix of the form

$$
C=\left(\begin{array}{ccc}
C_{11} & C_{12} & 0 \\
C_{21} & C_{22} & C_{23} \\
0 & C_{32} & C_{33}
\end{array}\right),
$$

may $C$ be written as $C=A \oplus_{k} B$, such that $A$ and $B$ are inverse-positive matrices?. We analyze this problem when $C_{22}$ is a real number or it is a matrix of size $k \times k$ with $k>1$.

First, we consider $k=1$. In this case, we obtain the following results.

Proposition 3 Let $A, B$ be the inverse-positive matrices

$$
A=\left(\begin{array}{cc}
A_{11} & 0 \\
a_{21}^{T} & a_{22}
\end{array}\right) \quad \text { and } \quad B=\left(\begin{array}{cc}
b_{11} & 0 \\
b_{21} & B_{22}
\end{array}\right)
$$

Then matrix $C=A \oplus_{1} B$ is inverse-positive.

Proof. The expressions of the inverses of $A$ and $B$ are

$$
A^{-1}=\left(\begin{array}{cc}
A_{11}^{-1} & 0 \\
-\frac{a_{21}^{T} A_{11}^{-1}}{a_{22}} & \frac{1}{a_{22}}
\end{array}\right), \quad B^{-1}=\left(\begin{array}{cc}
\frac{1}{b_{11}} & 0 \\
-\frac{B_{22}^{-1} b_{21}}{b_{11}} & B_{22}^{-1}
\end{array}\right)
$$

As $A$ and $B$ are inverse-positive matrices, blocks $A_{11}$ and $B_{22}$ are also inversepositive matrices, and the real numbers $a_{22}$ and $b_{11}$ are strictly positive.

The expression of the inverse of $C=A \oplus_{1} B$ is

$$
C^{-1}=\left(\begin{array}{ccc}
A_{11}^{-1} & 0 & 0 \\
-\frac{a_{21}^{T} A_{11}^{-1}}{a_{22}+b_{11}} & \frac{1}{a_{22}+b_{11}} & 0 \\
\frac{B_{22}^{-1} b_{21} a_{21}^{T} A_{11}^{-1}}{a_{22}+b_{11}} & -\frac{B_{22}^{-1} b_{21}}{a_{22}+b_{11}} & B_{22}^{-1}
\end{array}\right)
$$

It is not difficult to see that all the blocks of $C^{-1}$ are nonnegative matrices, so matrix $C$ is inverse-positive. 
Proposition 4 Let $C$ be an inverse-positive matrix of the form

$$
C=\left(\begin{array}{ccc}
C_{11} & 0 & 0 \\
c_{21}^{T} & c_{22} & 0 \\
0 & c_{32} & C_{33}
\end{array}\right)
$$

Then, $C$ can be expressed as $C=A \oplus_{1} B$ where $A$ and $B$ are inverse-positive matrices.

Proof. The expression of the inverse of $C$ is

$$
C^{-1}=\left(\begin{array}{ccc}
C_{11}^{-1} & 0 & 0 \\
-\frac{c_{21}^{T} C_{11}^{-1}}{c_{22}} & \frac{1}{c_{22}} & 0 \\
\frac{C_{33}^{-1} c_{32} c_{21}^{T} C_{11}^{-1}}{c_{22}} & -\frac{C_{33}^{-1} c_{32}}{c_{22}} & C_{33}^{-1}
\end{array}\right)
$$

Consider the following matrices

$$
A=\left(\begin{array}{cc}
C_{11} & 0 \\
c_{21}^{T} & x
\end{array}\right) \quad \text { and } B=\left(\begin{array}{cc}
y & 0 \\
c_{32} & C_{33}
\end{array}\right)
$$

As $c_{22}$ is a positive number, it can always be expressed as the sum of two positive numbers, so it must be accomplished that $x+y=c_{22}$ being $x$ and $y$ positive numbers. Consider, for example, $x=y=\frac{c_{22}}{2}$.

Matrices $A$ and $B$ are nonsingular and the expressions of their inverses are

$$
A^{-1}=\left(\begin{array}{cc}
C_{11}^{-1} & 0 \\
-2 \frac{c_{21}^{T} C_{11}^{-1}}{c_{22}} & \frac{2}{c_{22}}
\end{array}\right), \quad B^{-1}=\left(\begin{array}{cc}
\frac{2}{c_{22}} & 0 \\
-2 \frac{C_{33}^{-1} c_{32}}{c_{22}} & C_{33}^{-1}
\end{array}\right) .
$$

Bearing in mind that all the blocks of $C^{-1}$ are greater than or equal to zero, it is not difficult to notice that all the blocks of matrices $A^{-1}$ and $B^{-1}$ are also nonnegative, so matrices $A$ and $B$ are inverse-positive.

Proposition 5 Consider the inverse-positive matrices

$$
A=\left(\begin{array}{cc}
A_{11} & a_{12} \\
a_{21}^{T} & a_{22}
\end{array}\right) \quad \text { and } \quad B=\left(\begin{array}{ll}
b_{11} & b_{12}^{T} \\
b_{21} & B_{22}
\end{array}\right)
$$


where $a_{22}$ and $b_{11}$ are real numbers. If $A_{11}$ and $B_{22}$ are inverse-positive matrices, then $C=A \oplus_{1} B$ is an inverse-positive matrix.

Proof. After some algebraic operations, we obtain the following expressions of the inverses of matrices $A$ and $B$.

$$
\begin{aligned}
& A^{-1}=\frac{1}{a_{22}-a_{21}^{T} A_{11}^{-1} a_{12}}\left(\begin{array}{cc}
\left(a_{22}-a_{21}^{T} A_{11}^{-1} a_{12}\right) A_{11}^{-1}+A_{11}^{-1} a_{12} a_{21}^{T} A_{11}^{-1} & -A_{11}^{-1} a_{12} \\
-a_{21}^{T} A_{11}^{-1} & 1
\end{array}\right), \\
& B^{-1}=\frac{1}{b_{11}-b_{12}^{T} B_{22}^{-1} b_{21}}\left(\begin{array}{cc}
1 & -b_{12}^{T} B_{22}^{-1} \\
-B_{22}^{-1} b_{21} & \left(b_{11}-b_{12}^{T} B_{22}^{-1} b_{21}\right) B_{22}^{-1}+B_{22}^{-1} b_{21} b_{12}^{T} B_{22}^{-1}
\end{array}\right) .
\end{aligned}
$$

Using the Gauss method it is not difficult to obtain that determinants of matrices $A$ and $B$ are

$\operatorname{det}(A)=\left(a_{22}-a_{21}^{T} A_{11}^{-1} a_{12}\right) \operatorname{det}\left(A_{11}\right) \quad$ and $\quad \operatorname{det}(B)=\left(b_{11}-b_{12}^{T} B_{22}^{-1} b_{21}\right) \operatorname{det}\left(B_{22}\right)$, respectively.

Since $\operatorname{det}(A), \operatorname{det}(B), \operatorname{det}\left(A_{11}\right)$ and $\operatorname{det}\left(B_{22}\right)$ are nonzero, the real numbers $a_{22}-a_{21}^{T} A_{11}^{-1} a_{12}$ and $b_{11}-b_{12}^{T} B_{22}^{-1} b_{21}$ are also nonzero. Besides, if we look at the entries $(2,2)$ of $A^{-1}$ and $(1,1)$ of $B^{-1}$ we can conclude that the mentioned real numbers are strictly positive.

Calculating now the inverse of $C$ by solving the equation $C C^{-1}=I$, we obtain

$$
C^{-1}=\left(\begin{array}{ccc}
X & x_{12} & Z \\
x_{21}^{T} & \alpha & x_{23}^{T} \\
W & x_{32} & Y
\end{array}\right),
$$

where

$$
\begin{gathered}
X=A_{11}^{-1}\left(I-a_{12} x_{21}^{T}\right), \quad x_{12}=-\alpha A_{11}^{-1} a_{12}, \\
Z=-A_{11}^{-1} a_{12} x_{23}^{T}, \quad W=-B_{22}^{-1} b_{21} x_{21}^{T}, \\
x_{32}=-\alpha B_{22}^{-1} b_{21}, \quad Y=B_{22}^{-1}\left(I-b_{21} x_{23}^{T}\right), \\
x_{21}^{T}=\frac{-a_{21}^{T} A_{11}^{-1}}{a_{22}-a_{21}^{T} A_{11}^{-1} a_{12}+b_{11}-b_{12}^{T} B_{22}^{-1} b_{21}},
\end{gathered}
$$




$$
\begin{aligned}
\alpha & =\frac{1}{a_{22}-a_{21}^{T} A_{11}^{-1} a_{12}+b_{11}-b_{12}^{T} B_{22}^{-1} b_{21}}, \\
x_{23}^{T} & =\frac{-b_{12}^{T} B_{22}^{-1}}{a_{22}-a_{21}^{T} A_{11}^{-1} a_{12}+b_{11}-b_{12}^{T} B_{22}^{-1} b_{21}} .
\end{aligned}
$$

Bearing in mind that all the blocks of $A^{-1}$ and $B^{-1}$ are nonnegative we can conclude that all the blocks of $C^{-1}$ are greater than or equal to zero, so $C$ is an inverse-positive matrix.

When $A_{11}$ or $B_{22}$ are not inverse-positive matrices, the inverse-positivity of $C=A \oplus_{1} B$ is not guaranteed, as it can be seen in the next example.

Example 2 If we choose the inverse-positive matrix

$$
A=\left(\begin{array}{rrr}
0 & 0 & 1 \\
0 & 1 & -1 \\
1 & 0 & -1
\end{array}\right)
$$

with $A_{11}$ not inverse-positive, it is not difficult to check that matrix $C=A \oplus_{1} A$ is not inverse-positive.

Proposition 6 Consider the inverse-positive matrix

$$
C=\left(\begin{array}{ccc}
C_{11} & c_{12} & 0 \\
c_{21}^{T} & c_{22} & c_{23}^{T} \\
0 & c_{32} & C_{33}
\end{array}\right)
$$

where $C_{11}$ and $C_{33}$ are inverse-positive matrices. Then, $C$ can always be expressed as $C=A \oplus_{1} B$, where $A$ and $B$ are inverse-positive matrices.

Proof. The expression of $C^{-1}$ is

$$
C^{-1}=\frac{1}{\alpha}\left(\begin{array}{ccc}
\alpha C_{11}^{-1}+C_{11}^{-1} c_{12} c_{21}^{T} C_{11}^{-1} & -C_{11}^{-1} c_{12} & C_{11}^{-1} c_{12} c_{23}^{T} C_{33}^{-1} \\
-c_{21}^{T} C_{11}^{-1} & 1 & -c_{23}^{T} C_{33}^{-1} \\
C_{33}^{-1} c_{32} c_{21}^{T} C_{11}^{-1} & -C_{33}^{-1} c_{32} & \alpha C_{33}^{-1}+C_{33}^{-1} c_{32} c_{23}^{T} C_{33}^{-1}
\end{array}\right)
$$

where

$$
\alpha=c_{22}-c_{21}^{T} C_{11}^{-1} c_{12}-c_{23}^{T} C_{33}^{-1} c_{32}
$$


Using the Gauss method we obtain that determinant of matrix $C$ is

$$
\operatorname{det}(C)=\operatorname{det}\left(C_{11}\right) \operatorname{det}\left(C_{33}\right) \alpha \text {. }
$$

As this determinant cannot be zero, and it is known that $\operatorname{det}\left(C_{11}\right)$ and $\operatorname{det}\left(C_{33}\right)$ are not equal to zero, then $\alpha$ is not equal to zero. Besides, position $(2,2)$ of matrix $C^{-1}$ assures us that $\alpha$ is a positive real number.

Let $\varepsilon>0$ such that $\alpha-\varepsilon>0$, and consider the following matrices

$$
A=\left(\begin{array}{cc}
C_{11} & c_{12} \\
c_{21}^{T} & x
\end{array}\right) \quad \text { and } \quad B=\left(\begin{array}{cc}
y & c_{23}^{T} \\
c_{32} & C_{33}
\end{array}\right)
$$

where $x=c_{22}-c_{23}^{T} C_{33}^{-1} c_{32}-\varepsilon$ and $y=c_{22}-x$. Then, $x+y=c_{22}$ so $C=A \oplus_{1} B$.

The determinants of matrices $A$ and $B$, also obtained by the Gauss method, are given by

$\operatorname{det}(A)=\left(x-c_{21}^{T} C_{11}^{-1} c_{12}\right) \operatorname{det}\left(C_{11}\right)$ and $\operatorname{det}(B)=\left(y-c_{23}^{T} C_{33}^{-1} c_{32}\right) \operatorname{det}\left(C_{33}\right)$, respectively.

Note that

$$
x-c_{21}^{T} C_{11}^{-1} c_{12}=\alpha-\varepsilon>0
$$

and

$$
y-c_{23}^{T} C_{33}^{-1} c_{32}=\varepsilon>0 .
$$

We can then assert that $\operatorname{det}(A) \neq 0$ and $\operatorname{det}(B) \neq 0$. In other words, $A$ and $B$ are nonsingular matrices.

The expressions of the inverses of $A$ and $B$ are

$$
\begin{aligned}
A^{-1} & =\frac{1}{x-c_{21}^{T} C_{11}^{-1} c_{12}}\left(\begin{array}{cc}
\left(x-c_{21}^{T} C_{11}^{-1} c_{12}\right) C_{11}^{-1}+C_{11}^{-1} c_{12} c_{21}^{T} C_{11}^{-1} & -C_{11}^{-1} c_{12} \\
-c_{21}^{T} C_{11}^{-1} & 1
\end{array}\right), \\
B^{-1} & =\frac{1}{y-c_{23}^{T} C_{33}^{-1} c_{32}}\left(\begin{array}{cc}
1 & -c_{23}^{T} C_{33}^{-1} \\
-C_{33}^{-1} c_{32} & \left(y-c_{23}^{T} C_{33}^{-1} c_{32}\right) C_{33}^{-1}+C_{33}^{-1} c_{32} c_{23}^{T} C_{33}^{-1}
\end{array}\right) .
\end{aligned}
$$

Given that $C$ is an inverse-positive matrix, the entries of $C^{-1}$ in positions $(1,2),(2,1),(2,3)$ and $(3,2)$ are nonnegative. It follows, then, that the entries in positions $(1,2)$ and $(2,1)$ of $A^{-1}$ and of $B^{-1}$ are nonnegative. By multiplying the nonnegative entries $(1,2)$ and $(2,1)$ of matrix $C^{-1}$ we obtain $C_{11}^{-1} c_{12} c_{21}^{T} C_{11}^{-1}$, that must be also nonnegative. Besides, $C_{11}$ is an inverse-positive matrix, so matrix $A$ is inverse-positive. 
In a similar way, by multiplying the nonnegative entries $(3,2)$ and $(2,3)$ of matrix $C^{-1}$ we obtain $C_{33}^{-1} c_{32} c_{23}^{T} C_{33}^{-1}$, that must be also nonnegative. Besides, $C_{33}$ is an inverse-positive matrix, so matrix $B$ is also inverse-positive.

From now on we consider $k>1$. In this case, we obtain the following results.

Proposition 7 Consider the inverse-positive matrices of sizes $n_{1}$ and $n_{2}$ respectively,

$$
A=\left(\begin{array}{cc}
A_{11} & 0 \\
A_{21} & A_{22}
\end{array}\right) \quad \text { and } B=\left(\begin{array}{cc}
B_{11} & 0 \\
B_{21} & B_{22}
\end{array}\right),
$$

where $A_{22}$ and $B_{22}$ are $k \times k\left(1 \leq k \leq \min \left\{n_{1}, n_{2}\right\}\right)$. If matrix $H=A_{22}^{-1}+B_{11}^{-1}$ is inverse-positive, then matrix $C=A \oplus_{k} B$, of size $n=n_{1}+n_{2}-k$, is inverse-positive.

Proof. The expressions of the inverses of matrices $A$ and $B$ are

$$
A^{-1}=\left(\begin{array}{cc}
A_{11}^{-1} & 0 \\
-A_{22}^{-1} A_{21} A_{11}^{-1} & A_{22}^{-1}
\end{array}\right) \quad \text { and } \quad B^{-1}=\left(\begin{array}{cc}
B_{11}^{-1} & 0 \\
-B_{22}^{-1} B_{21} B_{11}^{-1} & B_{22}^{-1}
\end{array}\right) \text {. }
$$

In order to get the expression of the inverse of $C$ we use the next products of matrices.

$$
\left(\begin{array}{cc}
A^{-1} & 0 \\
0 & I_{n-n_{1}}
\end{array}\right) C\left(\begin{array}{cc}
I_{n-n_{2}} & 0 \\
0 & B^{-1}
\end{array}\right)=\left(\begin{array}{ccc}
I_{n-n_{2}} & 0 & 0 \\
0 & A_{22}^{-1}+B_{11}^{-1} & 0 \\
0 & 0 & I_{n-n_{1}}
\end{array}\right)
$$

Then

$$
C=\left(\begin{array}{ll}
A & 0 \\
0 & I_{n-n_{1}}
\end{array}\right)\left(\begin{array}{ccc}
I_{n-n_{2}} & 0 & 0 \\
0 & A_{22}^{-1}+B_{11}^{-1} & 0 \\
0 & 0 & I_{n-n_{1}}
\end{array}\right)\left(\begin{array}{cc}
I_{n-n_{2}} & 0 \\
0 & B
\end{array}\right) .
$$

And its inverse is

$$
C^{-1}=\left(\begin{array}{cc}
I_{n-n_{2}} & 0 \\
0 & B^{-1}
\end{array}\right)\left(\begin{array}{ccc}
I_{n-n_{2}} & 0 & 0 \\
0 & H^{-1} & 0 \\
0 & 0 & I_{n-n_{1}}
\end{array}\right)\left(\begin{array}{cc}
A^{-1} & 0 \\
0 & I_{n-n_{1}}
\end{array}\right),
$$


expression that can be written as

$$
C^{-1}=\left(\begin{array}{ccc}
A_{11}^{-1} & 0 & 0 \\
-B_{11}^{-1} H^{-1} A_{22}^{-1} A_{21} A_{11}^{-1} & B_{11}^{-1} H^{-1} A_{22}^{-1} & 0 \\
B_{22}^{-1} B_{21} B_{11}^{-1} H^{-1} A_{22}^{-1} A_{21} A_{11}^{-1} & -B_{22}^{-1} B_{21} B_{11}^{-1} H^{-1} A_{22}^{-1} & B_{22}^{-1}
\end{array}\right)
$$

Since $H$ is an inverse-positive matrix, it follows that $C$ is also inverse-positive.

The converse does not hold, as we can see in the next example.

Example 3 Matrices

$$
A=\left(\begin{array}{r|rr}
8 & 0 & 0 \\
\hline-1 & -1 & 2 \\
-1 & 3 & -1
\end{array}\right) \quad \text { and } B=\left(\begin{array}{rr|r}
-1 & 2 & 0 \\
3 & -1 & 0 \\
\hline-1 & -1 & 6
\end{array}\right)
$$

are inverse-positive, and matrix

$$
C=A \oplus_{2} B=\left(\begin{array}{r|rr|r}
8 & 0 & 0 & 0 \\
\hline-1 & -2 & 4 & 0 \\
-1 & 6 & -2 & 0 \\
\hline 0 & -1 & -1 & 6
\end{array}\right),
$$

is also inverse-positive, but matrix $H=A_{22}^{-1}+B_{11}^{-1}$ is not inverse-positive.

Now we consider again inverse-positive matrices partitioned as in Proposition 7. Let us denote $H^{\prime}=A_{22}+B_{11}$.

Proposition 8 Consider the inverse-positive matrices

$$
A=\left(\begin{array}{cc}
A_{11} & 0 \\
A_{21} & A_{22}
\end{array}\right) \quad \text { and } B=\left(\begin{array}{cc}
B_{11} & 0 \\
B_{21} & B_{22}
\end{array}\right) .
$$

If $A_{21} \leq 0, B_{21} \leq 0$ and matrix $H^{\prime}=A_{22}+B_{11}$ is inverse-positive, then $C=A \oplus_{k} B$ is inverse-positive.

Proof. The expressions of the inverses of $A$ and $B$ are

$$
A^{-1}=\left(\begin{array}{cc}
A_{11}^{-1} & 0 \\
-A_{22}^{-1} A_{21} A_{11}^{-1} & A_{22}^{-1}
\end{array}\right) \quad \text { and } \quad B^{-1}=\left(\begin{array}{cc}
B_{11}^{-1} & 0 \\
-B_{22}^{-1} B_{21} B_{11}^{-1} & B_{22}^{-1}
\end{array}\right)
$$


and the expression of the inverse of $C$ is

$$
C^{-1}=\left(\begin{array}{ccc}
A_{11}^{-1} & 0 & 0 \\
-H^{\prime-1} A_{21} A_{11}^{-1} & H^{\prime-1} & 0 \\
B_{22}^{-1} B_{21} H^{\prime-1} A_{21} A_{11}^{-1} & -B_{22}^{-1} B_{21} H^{\prime-1} & B_{22}^{-1}
\end{array}\right)
$$

If $A_{21} \leq 0, B_{12} \leq 0$ and $H^{\prime}$ is inverse-positive, it is not difficult to prove that $C$ is inverse-positive.

Proposition 9 Consider the inverse-positive matrix

$$
C=\left(\begin{array}{ccc}
C_{11} & 0 & 0 \\
C_{21} & C_{22} & 0 \\
0 & C_{32} & C_{33}
\end{array}\right)
$$

where $C_{22}$ is of size $k \times k$. Then $C$ can be expressed as $C=A \oplus_{k} B$, where $A$ and $B$ are inverse-positive matrices.

Proof. Suppose that matrices $A$ and $B$ have the form

$$
A=\left(\begin{array}{cc}
C_{11} & 0 \\
C_{21} & A_{22}
\end{array}\right) \quad \text { and } B=\left(\begin{array}{cc}
B_{11} & 0 \\
C_{32} & C_{33}
\end{array}\right)
$$

where $A_{22}=B_{11}=\frac{C_{22}}{2}$. Obviously, $A_{22}+B_{11}=C_{22}$, so $C=A \oplus_{k} B$.

The determinants of matrices $A$ and $B$ are

$$
\operatorname{det}(A)=\left(\frac{1}{2}\right)^{k} \operatorname{det}\left(C_{11}\right) \operatorname{det}\left(C_{22}\right) \quad \text { and } \quad \operatorname{det}(B)=\left(\frac{1}{2}\right)^{k} \operatorname{det}\left(C_{22}\right) \operatorname{det}\left(C_{33}\right),
$$

respectively, and they are not equal to zero because the determinant of matrix $C$ is

$$
\operatorname{det}(C)=\operatorname{det}\left(C_{11}\right) \operatorname{det}\left(C_{22}\right) \operatorname{det}\left(C_{33}\right)
$$

and it is not equal to zero, so matrices $A$ and $B$ are nonsingular and the expression of their inverses is

$$
A^{-1}=\left(\begin{array}{cc}
C_{11}^{-1} & 0 \\
-2 C_{22}^{-1} C_{21} C_{11}^{-1} & 2 C_{22}^{-1}
\end{array}\right) \quad \text { and } \quad B^{-1}=\left(\begin{array}{cc}
2 C_{22}^{-1} & 0 \\
-2 C_{33}^{-1} C_{32} C_{22}^{-1} & C_{33}^{-1}
\end{array}\right)
$$


while the expression of the inverse of $C$ is

$$
C^{-1}=\left(\begin{array}{ccc}
C_{11}^{-1} & 0 & 0 \\
-C_{22}^{-1} C_{21} C_{11}^{-1} & C_{22}^{-1} & 0 \\
C_{33}^{-1} C_{32} C_{22}^{-1} C_{21} C_{11}^{-1} & -C_{33}^{-1} C_{32} C_{22}^{-1} & C_{33}^{-1}
\end{array}\right) .
$$

Given that $C$ is an inverse-positive matrix, it follows that $A$ and $B$ are inversepositive matrices.

Proposition 10 Consider the inverse-positive matrices

$$
A=\left(\begin{array}{cc}
A_{11} & 0 \\
A_{21} & A_{22}
\end{array}\right) \quad \text { and } B=\left(\begin{array}{cc}
B_{11} & B_{12} \\
0 & B_{22}
\end{array}\right)
$$

with $A_{22}$ and $B_{22}$ blocks of size $k \times k$. If $A_{21} \leq 0, B_{12} \leq 0$ and matrix $H^{\prime}=$ $A_{22}+B_{11}$ is inverse-positive, then $C=A \oplus_{k} B$ is inverse-positive.

Proof. The inverses of $A$ and $B$ are

$$
A^{-1}=\left(\begin{array}{cc}
A_{11}^{-1} & 0 \\
-A_{22}^{-1} A_{21} A_{11}^{-1} & A_{22}^{-1}
\end{array}\right) \quad \text { and } \quad B^{-1}=\left(\begin{array}{cc}
B_{11}^{-1} & -B_{11}^{-1} B_{12} B_{22}^{-1} \\
0 & B_{22}^{-1}
\end{array}\right)
$$

and the inverse of $C$ is

$$
C^{-1}=\left(\begin{array}{ccc}
A_{11}^{-1} & 0 & 0 \\
-H^{\prime-1} A_{21} A_{11}^{-1} & H^{\prime-1} & -H^{\prime-1} B_{12} B_{22}^{-1} \\
0 & 0 & B_{22}^{-1}
\end{array}\right) .
$$

Since $A_{21} \leq 0, B_{12} \leq 0$ and $H^{\prime}$ is inverse-positive, we can assert that $C$ is an inverse-positive matrix.

Proposition 11 Let $C$ be an inverse-positive matrix of the following form

$$
C=\left(\begin{array}{ccc}
C_{11} & 0 & 0 \\
C_{21} & C_{22} & C_{23} \\
0 & 0 & C_{33}
\end{array}\right)
$$

where $C_{22}$ is a $k \times k$ block. Then, $C$ can be expressed as $C=A \oplus_{k} B$, where $A$ and $B$ are inverse-positive matrices. 
Proof. The expression of the inverse of $C$ is

$$
C^{-1}=\left(\begin{array}{ccc}
C_{11}^{-1} & 0 & 0 \\
-C_{22}^{-1} C_{21} C_{11}^{-1} & C_{22}^{-1} & -C_{22}^{-1} C_{23} C_{33}^{-1} \\
0 & 0 & C_{33}^{-1}
\end{array}\right) .
$$

Let

$$
A=\left(\begin{array}{cc}
C_{11} & 0 \\
C_{21} & A_{22}
\end{array}\right) \quad \text { and } B=\left(\begin{array}{cc}
B_{11} & C_{23} \\
0 & C_{33}
\end{array}\right),
$$

where $A_{22}=B_{11}=\frac{C_{22}}{2}$. It is clear that $A_{22}+B_{11}=C_{22}$ and $C=A \oplus_{k} B$. We have

$$
\operatorname{det}(A)=\left(\frac{1}{2}\right)^{k} \operatorname{det}\left(C_{11}\right) \operatorname{det}\left(C_{22}\right) \quad \text { and } \quad \operatorname{det}(B)=\left(\frac{1}{2}\right)^{k} \operatorname{det}\left(C_{22}\right) \operatorname{det}\left(C_{33}\right) .
$$

These determinants are nonzero since

$$
\operatorname{det}(C)=\operatorname{det}\left(C_{11}\right) \operatorname{det}\left(C_{22}\right) \operatorname{det}\left(C_{33}\right) \neq 0 \text {. }
$$

Therefore, $A$ and $B$ are nonsingular. Moreover,

$$
A^{-1}=\left(\begin{array}{cc}
C_{11}^{-1} & 0 \\
-2 C_{22}^{-1} C_{21} C_{11}^{-1} & 2 C_{22}^{-1}
\end{array}\right) \quad \text { and } \quad B^{-1}=\left(\begin{array}{cc}
2 C_{22}^{-1} & -2 C_{22}^{-1} C_{23} C_{33}^{-1} \\
0 & C_{33}^{-1}
\end{array}\right) .
$$

It is not difficult to see that matrices $A$ and $B$ are inverse-positive.

The general case $C=A \oplus_{k} B$, where $A$ and $B$ have the form

$$
A=\left(\begin{array}{ll}
A_{11} & A_{12} \\
A_{21} & A_{22}
\end{array}\right) \quad \text { and } \quad B=\left(\begin{array}{cc}
B_{11} & B_{12} \\
B_{21} & B_{22}
\end{array}\right),
$$

and the converse problem are still open problems. In general, the two questions analyzed in this paper about inverse-positivity in sub-direct sums have a negative answer. For the first question we can see Example 1 and for the second one we can see the following example.

Example 4 Matrix

$$
C=\left(\begin{array}{r|rr|r}
0 & 0 & 0 & 1 \\
\hline 1 & 0 & -1 & -1 \\
-1 & 1 & 1 & 0 \\
\hline 0 & 0 & 1 & 0
\end{array}\right),
$$


is inverse-positive, but it cannot be expressed as $A \oplus_{2} B$ for any square matrices $A$ and $B$ of size $3 \times 3$.

\section{Acknowledgement}

The authors would like to thank the referee for the valuable comments and for the suggestions to improve the readability of the paper.

\section{References}

[1] A. Berman, R. Plemmons, Nonnegative matrices in the Mathematical Sciences, SIAM, Philadelphia, 1994

[2] M. Fiedler, Characterizations of sign patterns of inverse-positive matrices, Linear Algebra ans its Applications, 40 (1981) 237-245.

[3] J.E. Peris, A new characterization of inverse-positive matrices, Linear Algebra ans its Applications, 154-156 (1991) 45-58.

[4] T. Fujimoto, R.R. Ranade, Two characterizations on inverse-positive matrices: The Hawkins-Simon condition and the Chatelier-Braun principle, ELA 11 (2004) 59-65.

[5] D. Hawkins, H.A. Simon, Some conditions of Macroeconomic stability, Econometrica, 17 (1949) 245-248.

[6] A. Greenbaum, Iterative methods for solving nonlinear systems, SIAM, Philadelphia, 1997.

[7] R. Precup, Two positive nontrivial solutions for a class of semilinear elliptic variational systems, Journal of Mathematical Analysis and Applications, 373 (2011) 138-146.

[8] C.M. da Fonseca, On the eigenvalues od some tridiagonal matrices, Journal of Computational and Applied Mathematics, 200 (2007) 283-286.

[9] T. Fujimoto, J.A. Silva and A. Villar, Nonlinear generalizations of theorems on Inverse-Positive Matrices, Advances in Mathematical Economics, 5 (2003) 55-63.

[10] S. Fallat, C. Johnson, Sub-direct sums and positivity classes of matrices, Linear Algebra and its Applications, 288 (1999) 149-173.

[11] A. Toselli, O. Widlund, Domain decomposition methods: Algorithms and Theory, Series in Computational Mathematics, Vol. 34, Springer, New York, 2005.

[12] R. Nabben, D.B. Szyld, Convergence theory of restricted multiplicative Schwarz methods, SIAM Journal Numerical Analysis, 40 (2003) 2318-2336. 